\title{
Energy Aware Communication in the Smart Grid
}

\author{
Zoya Pourmirza and John M. Brooke \\ School of Computer Science, The University of Manchester, Manchester, U.K.
}

Keywords: Smart Grid, NAN, Energy Efficiency, ICT Architecture, Optimal Network Topology, Data Reduction.

\begin{abstract}
Recently some countries have investigated the transformation of their existing power grid to the so-called Smart Grid. A Smart Grid adds a communication network, which is the integration of a monitoring and control network, to the power network. In this research we have developed and implemented a communication network architecture for the neighbourhood sub-Grid level of the electrical network where monitoring has not previously been deployed. Since energy efficiency has been identified as one of the major limitations of such networks we have utilized a number of different techniques to tackle this problem. As such, we analyse the optimal topology of network for collecting and transmitting data to the local control unit for applying finer-grained control. Also, we have developed a data reduction algorithm suitable for Smart Grid applications, which can significantly improve the energy efficiency of the communication network by minimizing the communication energy cost and optimizing the network resource consumption while maintaining the integrity and quality of data. To the best of our knowledge, our work is one of the very first efforts to propose an energy efficient ICT architecture, combining power grid objectives, real data characteristics, and application-aware considerations.
\end{abstract}

\section{INTRODUCTION}

In the recent years scientists have identified a number of problems associated with the conventional power grid and tried to tackle them. This has led to the birth of the concept of a Smart Grid. According to the US Department of Energy (DOE) (Miller et al., 2008) the Smart Grid should contain the following characteristics; self-healing, consumer friendly, reliable with good power quality, resistant to cyber-attack, and facilitating new service and markets. To achieve these features necessitates the incorporation of the Information and Communication Technology (ICT) along with the power network.

Deploying a large number of monitoring devices in the Smart Grid environment that transmit huge volume of data will saturate the device resources and consumes their energy. Some of the key constraints of wireless sensor devices are their limited resources such as memory, battery and processing power. Since battery technology used in the sensor network has a slower development rate than both the sensor device technology and the processor technology (Miao et al., 2009), the energy constraint problem has emerged as one of the main limitations of these network when wireless communication is proposed. In such networks difficulties arise when the deployed sensors are short on power, thus a specific area of the grid is no longer being monitored at a sufficient rate, which will lead to incorrect decision making by the control mechanisms and operators of the grid.

In this paper we will summarize and integrate different aspects of an experimental Smart Grid project taking place in the University of Manchester. We will briefly discuss our proposed ICT architecture, which involves the integration of various monitoring devices, storage and control units for the neighbourhood area network (NAN) of the ICT network within the Smart Grid. Implementing our proposed architecture in the real environment rather than solely in a simulation-based environment, enables us to identify a number of drawbacks associated with such networks. One of the major problems which we will discuss in this paper is the energy efficiency consideration of the communication network. These limitations necessitate development of the techniques to consume sensor resources more efficiently to achieve better quality of the network, longer lifetime and time between maintenance sessions.

Observing that $50 \%$ of energy is spent during the 
data transmission process, we realize that it is not practical to broadcast full resolution data over long distances. Therefore, we investigated how to configure the communication network of the wireless monitoring devices and also how to apply the data reduction techniques to decrease the size of transmitted file before transmission process. These result in more effective utilization of available resources and offer better energy efficiency to the system.

\section{PROPOSED ICT ARCHITECTURE FOR SMART GRIDS}

In (Pourmirza and Brooke, 2013c) we proposed two related architectural structures and discussed our experimental results for the ICT infrastructure of an urban environment in the Smart Grid. These two architectures are: - a communication network architecture and a software architecture. In this section we summarize the communication architecture and its constituent components. In the next section we will demonstrate the techniques we have used to provide energy efficient communication architecture specially designed for the NAN of the Smart Grid.

The proposed architecture is a modular architecture that integrates the peer-to-peer hierarchical architectures, tailored to hybrid communication technologies for transmitting data. It is being implemented on the medium voltage power network substation $6.6 \mathrm{kV}$ of the University of Manchester campus which owns its own distribution grid. This allows us to validate our architectural designs on real equipment, real data, and input from experts in power engineering. This architecture (figure 1) comprises five layers that cooperate to offer four main functions of monitoring, data movement, data storage and control. Our proposed architecture offers modularity, scalability, fault tolerance, energy efficiency and future proofing against changes in networking technology. Each of these characteristics are discussed in details in (Pourmirza and Brooke, 2013c).

The first layer in the figure 1 consists of smart meters as monitoring devices, which are situated in all the buildings of the University of Manchester. They are responsible for monitoring building level data that provide information about power usage and permit the management of the power generation and consumption. The information collected at this level is valuable for prosumers (producer-consumer), because by integrating such data with data on realtime energy prices we can offer effective demand response control.

The second layer comprises hundreds of sensors located in the street areas that are responsible for monitoring environmental data such as temperature, light, and humidity. The data collected at this level is essential for understanding the response of demand on the electrical system to variables such as number of people, weather, temperature, humidity and so on. It also can help in controlling the power grid by delivering data that can be used to anticipate demand and improve control actions.

The second layer itself is divided into two sublayers. This division is due to the energy constraints of the Wireless Sensor Network (WSN). Consequently we have utilized cluster based communication as a method of communication instead of direct communication as a more energy efficient data transmission technique (Abbasi and Younis, 2007). In order to evaluate the WSN at this level, we extended the TinyDB (MADDEN et al., 2005) (WSN query processing engine) by adding a Smart Grid component to it. The extension to TinyDB enables us to run and test our prototype implementation in the laboratory-based environment as well as in a real physical environment.

The third layer comprises the monitoring and control devices situated in $6.6 \mathrm{kV}$ substations in our test bed. They are responsible for monitoring three phase voltages, currents, active power, power factors, voltage's spectra (eight channels for each phase) and current spectra (eight channels) and frequency. These data are useful for fault identification, power quality analysis, and many other applications. This layer is also divided into three sub-layers: namely a reconfigurable real-time control and acquisition system called Compact RIO (cRIO), data storage, and a control unit which is a program responsible for applying control over the substations only, that is called LabVIEW (LabVIEW, 2007). The next layer (fourth) is the database (DB) layer responsible for storing data received from the layers below.

The final layer is the Neighbourhood Control Unit (NCU) which extracts data from the database layer or directly from the sensors. We have developed a Geographical Information System (GIS) enhanced display tool (Pourmirza and Brooke, 2013b) for this layer which is beneficial for visualizing the live/historical data on a map view, and for tracking and identifying the faulty part of the system in advance. 


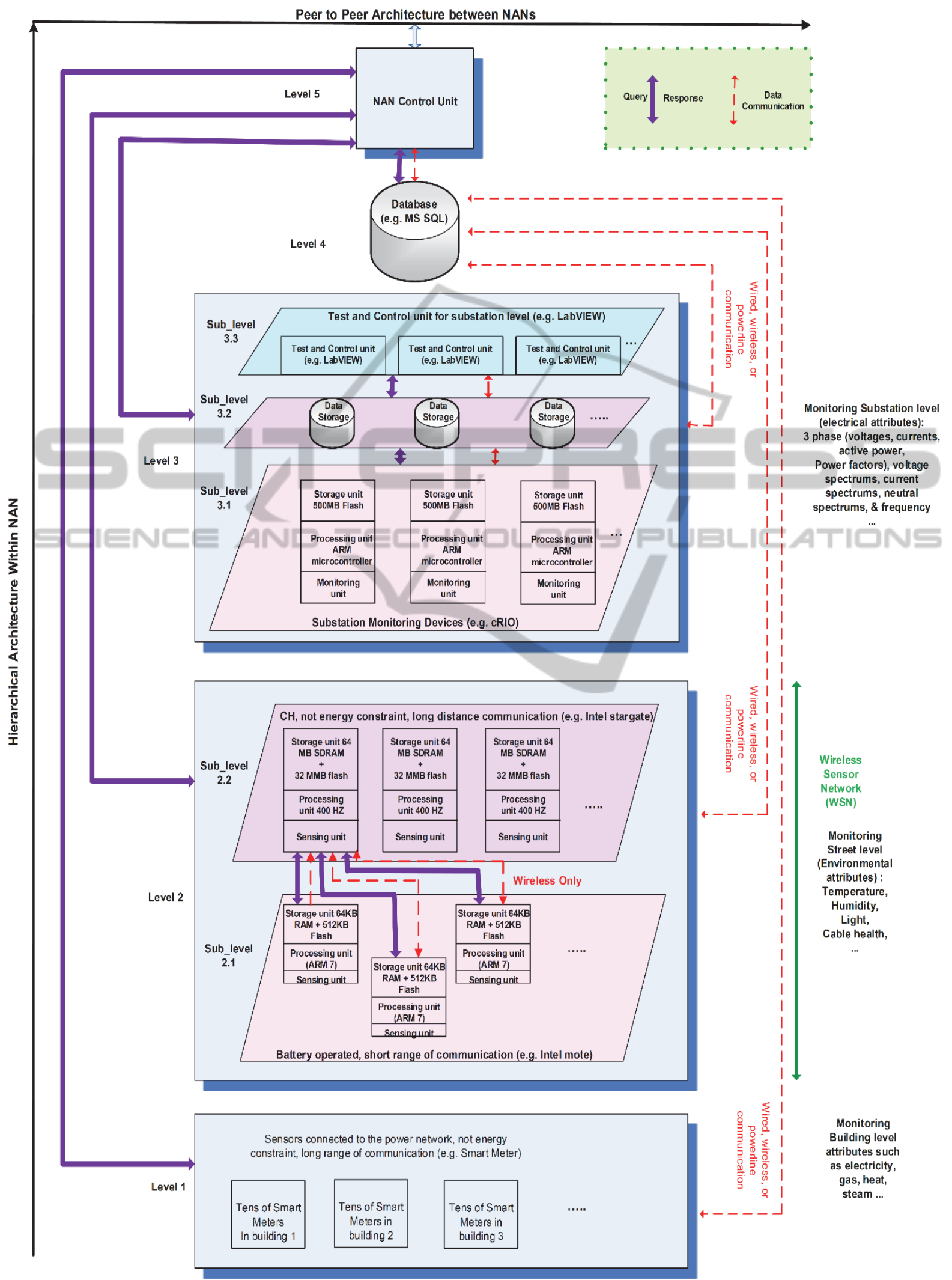

Figure 1: An ICT architecture for the NAN in the Smart Grid (Pourmirza and Brooke, 2013c). 


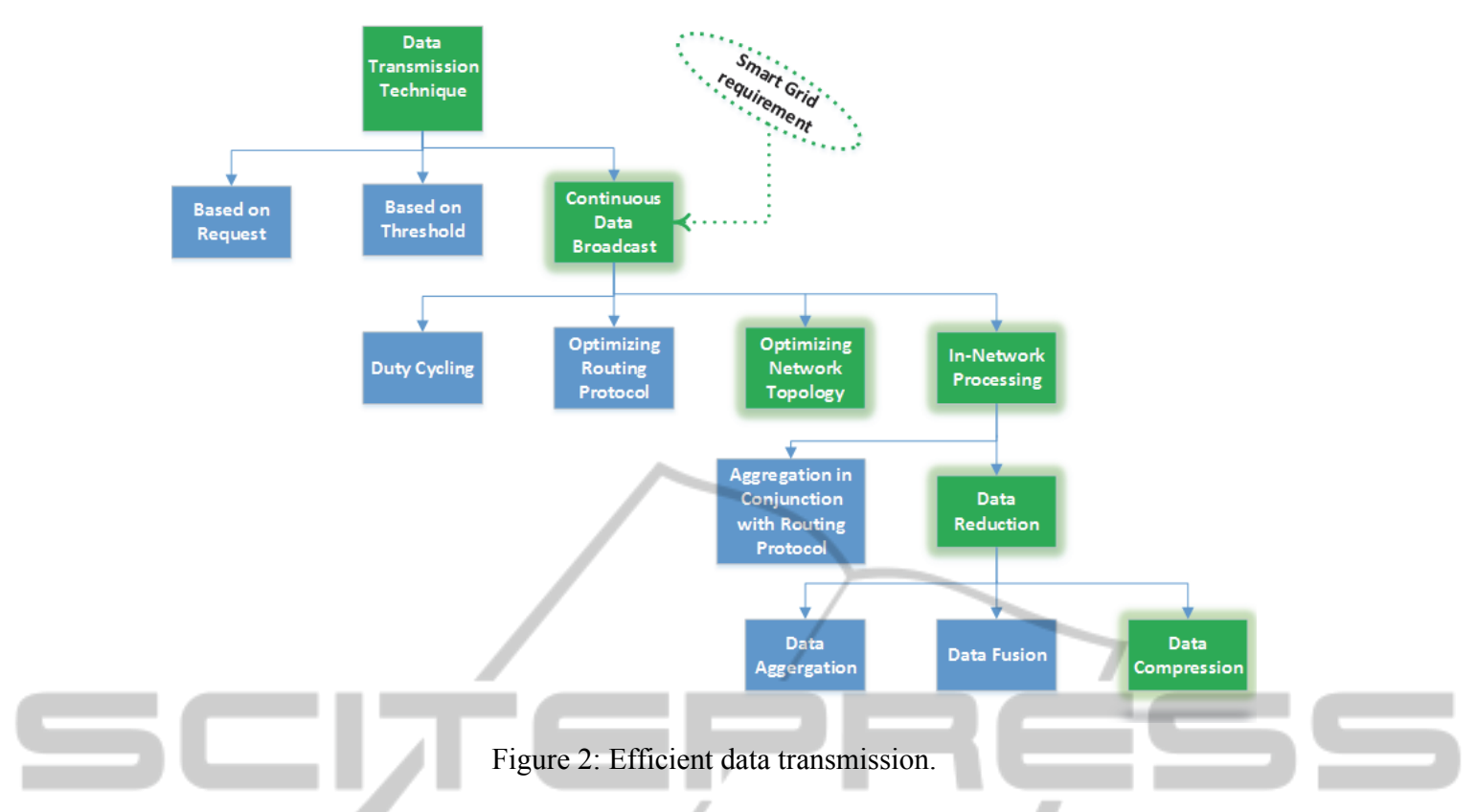

In an urban environment multiple NAN systems should join to form a higher sub-grid network. Therefore, we have added another layer of communication over the top of the architecture to enable these systems to communicate and coordinate their control actions.

\section{TECHNIQUES TO PROVIDE ENERGY EFFICIENT DATA TRANSMISSION}

We have classified the data transmission technique, which is the technique devices use to transfer their data to the NCU, into three different categories. The first category is used when sensors transmit their data after receiving requests from the sink. The second category is when sensors send data indicating that a threshold condition is violated or when an emergency situation has occurred. The third category is when sensors collect data and broadcast them continuously. Although the first and second category are more energy efficient methods of data transmission, because data are being shipped with lower frequency or only occasionally, our NAN monitoring network in the Smart Grid also necessitates the third category, because electrical engineers want to monitor data collected from the grid at all times. Thus full data collection is needed in such networks.

Our survey of the literature reveals that the energy efficient radio communication can be accomplished through different means, such as; duty cycling, optimizing the routing algorithm, optimizing the network topology, and in-network processing. Duty cycling can be achieved through scheduling the sleep/wakeup program of sensors (Anastasi et al., 2006), which is not, however, an appropriate technique in our context, since our sensors are continuously sensing and sending data without going back to sleep mode. Optimizing the routing algorithm can be accomplished by developing a multi-hop routing algorithm that can identify the next optimal hop to route the message to the sink. In our network design we assume we have direct communication instead of multi-hop communication; therefore, optimizing the routing protocol is out of the scope of this research. Optimizing the network topology can be achieved through managing the communication distance, which is the first technique we have utilized in our research. Finally in-network processing can be classified in two classes. The first class is the data aggregation techniques being implemented in conjunction with WSN routing protocols. A survey of data aggregation techniques in WSNs (Thangaraj and Ponmalar, 2011) has introduced and analysed several such data aggregation protocols. The second class of in-network processing methods is called data reduction, which is performed by implementing data reduction algorithms to reduce the communication cost by minimizing the size of transmitted data. Applying data reduction will result in efficient bandwidth utilization and also power saving caused by data transmission, which will increase the network lifetime (Naoto and Shahram, 2005). The technique used in this research to offer 
energy efficiency to the communication network belongs purely to the second class of in-network processing, i.e. the data reduction method.

Data reduction itself can be classified into data aggregation, data fusion, and in-network data compression. Data aggregation is useful when the goal is to reduce the communication overhead and cost. This method will reduce the message size by utilizing one of the aggregation functions such as Min, Max, Sum, and Average (Tan et al., 2007).

Data fusion is a more elegant method in comparison with the data aggregation. In this method various unreliable data will be combined to eliminate the related noise and produce a more accurate signal (Abdelgawad and Bayoumi, 2012). Data compression can be described as the procedure of processing raw data into a condensed structure rather than its original format. One of the challenges in the data compression technique is the accuracy of the decompression algorithm while reconstructing the data. The data compression is usually used in the applications where the full data collection is required. Since electrical engineers working on the Smart Grid applications are still evaluating the data we do not use fusion or aggregation. Thus, our data reduction algorithm is purely a compression method which keeps the quality and integrity of data. Figure 2 demonstrates the categories and subcategories of efficient data transmission, and highlights (in green) the contribution of our research to this area.

\subsection{Optimizing the Network Topology}

As discussed earlier, the first technique we have used to provide more energy efficient architecture is to optimize the WSN topology. In this section we focus on the second layer of the architecture and identify the optimal topology of the WSN by analysing the optimal number of clusters for specific application in our test bed. However, it should be noted that these results may vary due to changes in the networking topology, communication protocol, and Quality of Service (QOS) requirements. As shown in figure 3 the second layer of the architecture is going to be deployed in the University of Manchester sub-Grid. The university campus is embedded in a city, containing streets and road. The whole campus is connected by rectangular grid.

Since we are dealing with an urban area the sensors are located at fixed locations which, to a first approximation, can be located on a rectangular grid reflecting the pattern of the urban streets, as shown in figure 3 .

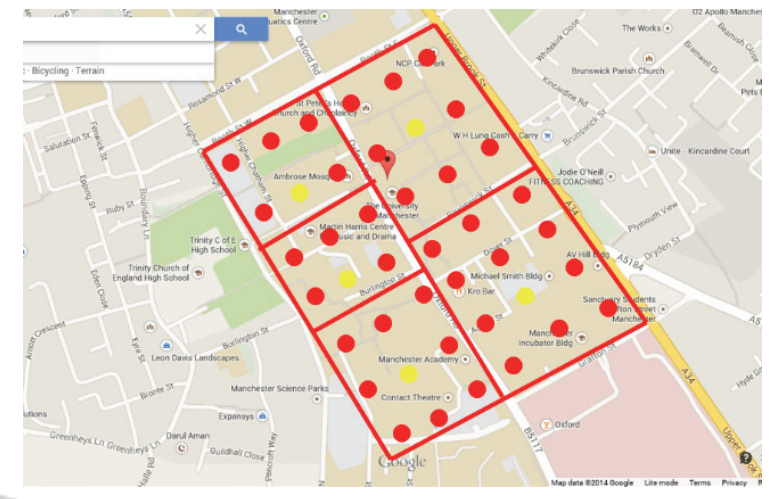

Figure 3: The University of Manchester campus, representing a rectangular grid, being divided into clusters. The red dots show normal sensors, while yellow dots are considered to be a cluster head.

In order to obtain the most energy efficient topology of a grid we must find the optimal number of cluster. Therefore we divide the network into various number of clusters and maintain a fixed number of sensors. Then we start simulating each of these networks by varying the number of sensors in the clusters, cluster shapes and locations for 12 different configurations, all of which preserve the number of clusters. We have considered different configurations, because in real projects it is not always possible to deploy the nodes in the most optimal topology, thus we estimate the variance. Figure 4 shows total energy consumption against the number of clusters. The curve demonstrates a minimum at 8 clusters. The result that the minimum occurs at 8 clusters is a function of the total size of our rectangular grid $(10 \times 10)$ and the amount of energy consumed by the $\mathrm{CHs}$; however, the methods could be used on grids of arbitrary size and CHs. More details are described in (Pourmirza and Brooke, 2013d).

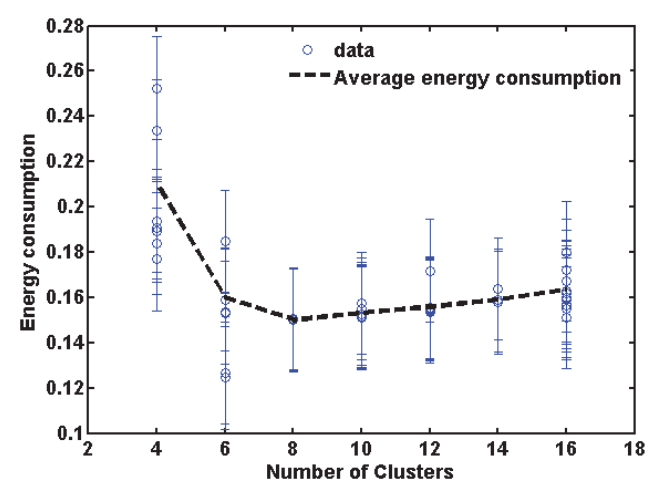

Figure 4: Energy cost analysis of a grid with different number of clusters. 
Finally our experiments reveal that the two other factors that affect the energy consumption of a system are the shape and location of the clusters. We observe that, if the clusters are rectangular, then the best result is when the rectangles of the grid are square. Moreover if we consider different size of clusters in a network, by locating the smaller clusters near the data sink, and bigger clusters farther from the sink, we can also improve energy efficiency of the WSN.

\subsection{Developed Data Reduction Algorithm}

The second technique utilized in this research to offer communication energy efficiency is to develop a data reduction algorithm. The exponential increase of the number of monitoring devices in the Smart Grid will lead to an explosion in the data volume. The recent evidence (Allalouf et al., 2011); (IBM, 2012); (McNamara and Meynardi, 2010) has confirmed this - conclusion. As - current communication methods deployed in electrical Grids are not yet prepared to manage such volume of data, we have to start developing new methods and techniques to ease the transmission and storage of such data. An effective data reduction technique would be an effective approach in this context.

Previously, in (Pourmirza and Brooke, 2013a) we proposed a data reduction algorithm for data that is sampled at a higher rate than the rate at which successive values change significantly. Recently, we have improved our data reduction algorithm to further compress the data before transmission. Our first data reduction algorithm is called DRACO-1 (Data Reduction Algorithm for Correlated data) and the improved version is called DRACO-2. In the rest of this paper we will summarize DRACO-1, introduce DRACO-2 and demonstrate our experimental results.

In the Smart Grid applications, where the metering devices collect data with a high acquisition rate and transmit them to the NAN control unit, a great degree of data correlation occurs. Taking this fact into consideration, we have developed a data reduction algorithm (DRACO-1) that discards the redundant bits by applying XOR on each two consecutive measured values, and transmits the changing bits only. The changed bits are a small portion of the binary representation which will be converted to digit-based representation before transmission. DRACO-1 is discussed in detail in (Pourmirza and Brooke, 2013a). This algorithm can improve the energy efficiency of the communication network by transmitting smaller value of data while keeping the data integrity.

The difference between the DRACO-1 (method 1) and DRACO-2 (method 2) is that on the transmitter side, after applying XOR and converting the binary representation back to digit-based representation, if any consecutive values are similar, DRACO-2 will only send one instance of that value together with the number of repetition times. Although DRACO-2 is not as stable and general as DRACO-1, DRACO-2 is very helpful in compressing high volume data with strong correlations (such as frequency and voltage data) and in these cases it can perform better than DRACO-1.

In order to evaluate the compression efficiency of both DRACOs, and also assess their performance on different hours of a day, and check the behavior of the electrical grid during the peak hours and nonpeak hours, we have examined both DRACOs on 24 hours of real data (these data are collected from 8:00 $24^{\text {th }}$ April 2013 to 8:00 25 $5^{\text {th }}$ April 2013 with frequency of $1 \mathrm{~Hz}$ from a $6.6 \mathrm{kV}$ substations in our test bed). Figure 5 compares DRACO-1 (blue line) and DRACO-2 (red line) using the voltage data. It shows that DRACO-2 gains a higher compression ratio and is a more efficient algorithm for voltage compression. Moreover, it reveals that after 16:00 (4 p.m.) we can gradually achieve better compression (over $89 \%$ ) ratio until 1:00, which means the electrical grid is steadier and as the result the data correlation is higher during this period of time. Checking the load profile of the system has confirmed our results.

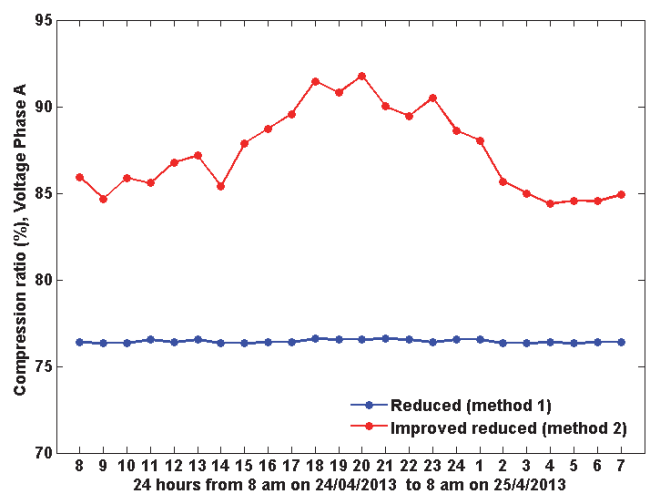

Figure 5: 24 hours of compressed voltage data.

Additionally, another experiment has been designed to assess the effect of various sampling rate on the efficiency of our data reduction algorithm. We have examined the data being logged with different frequency. Figure 6 shows that, as the 
frequency of data acquisition rate increase, the original size of the data will increase, which is simple to understand. However, as we start to sample more frequently the gap between the original data size and the reduced data will grow as well. Thus, with higher sampling rate we are transmitting more information about the grid, and with the use of the DRACOs we can send this information more efficiently. This result confirms the observation that when data are being sampled at a faster rate, the correlation between each two consecutive value is higher, and DRACO performs best on data with stronger correlations. Moreover, we conclude that the size of the reduced data file (DRACO-1) with data being logged once every second is roughly equal to the size of original data being logged once every 3 seconds. This result is beneficial in terms of bandwidth utilization and hence energy consumption.

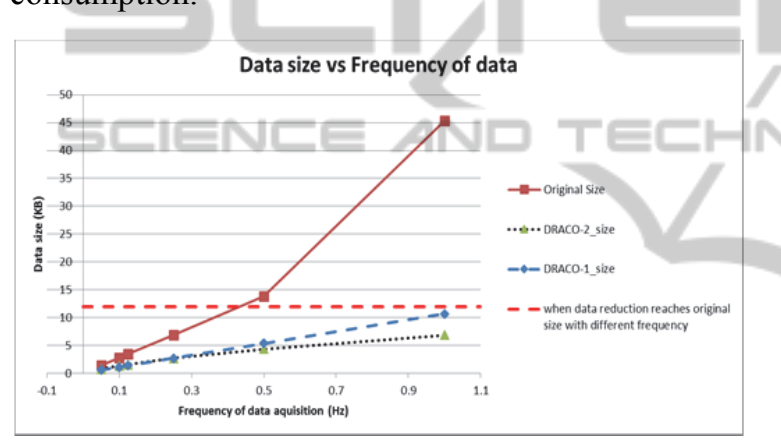

Figure 6: Data acquisition rate evaluation.

\section{CONCLUSIONS}

In this paper we present a practical energy efficient ICT architecture for the NAN in the Smart Grid. Although we had previously discussed about different aspects of this research separately, this is the first time that all these finding are integrated to present a completed project.

At the beginning of this research we proposed a communication network architecture and analysed its design in terms of offering energy efficiency for a local control system. Then we evaluated the techniques to offer more energy efficient data transmission. As the result we follow two approaches, optimizing the network topology and applying a data reduction technique at various level of the architecture. Regarding the optimization of the network topology, we present our experimental results that the number of clusters, their shapes and the way the clusters are geographically grouped are all important to offer deeper a minimum of energy consumption. Then we presented an updated version of our data reduction algorithm (DRACO.) We confirmed experimentally that the data from the NAN of the Smart Grid conformed to the pattern required by both DRACOs. An important part of this work is that we have been able to validate and test DRACO on data, which are produced at a very high sampling rate. This is useful in terms of identifying key changes in behaviour of electrical systems. One significant contribution of this research is that the algorithm can reduce the power consumption and improve the overall energy efficiency of the communication network in the proposed architecture. Finally, it can provide an efficient flow of information by reducing data traffic and accelerating data transmission speed. Since, in such networks, the bottleneck is caused by the fact that thousands of sensors are sending their data to the central point, in some cases by applying DRACO and reducing data roughly by an order of magnitude (to base 10) we can reduce the risk of bottleneck.

In future we will monitor the performance of DRACO over extended period of time to estimate how much energy and storage could be saved in an ICT network with this technique.

\section{ACKNOWLEDGEMENTS}

We are thankful to Professor Ian Cotton and $\mathrm{Mr}$ Peter R. Green in the school of Electrical and Electronic Engineering in the University of Manchester for providing experimental data and their valuable feedback.

\section{REFERENCES}

What is NI LabVIEW? [Online]. National Instruments. Available: http://www.ni.com/labview/whatis/ [Accessed 02/07/2011].

Abbasi, A. A. \& Younis, M. (2007) A survey on clustering algorithms for wireless sensor networks. Computer Communication, 30(14-15), 2826-2841.

Abdelgawad, A. \& Bayoumi, M. (2012) Data Fusion in WSN. Resource-Aware Data Fusion Algorithms for Wireless Sensor Networks. Springer.

Allalouf, M., Gershinsky, G., Lewin-Eytan, L. \& Naor, J. (2011) Data-quality-aware volume reduction in smart grid networks. In: IEEE International Conference on Smart Grid Communications (SmartGridComm) 1720 October 2011 Brussels, Belgium. IEEE, 120-125.

Anastasi, G., Conti, M., Di Francesco, M. \& Passarella, A. (2006) How to prolong the lifetime of wireless sensor networks. Valencia, CA: American Scientific 
Publishers.

IBM. (2012) Managing big data for smart grids and smart meters. Information Management. USA.

LabVIEW. (2007) LabVIEW Fundamentals. Austin, Texas: National Instruments Corporation.

MADDEN, S. R., FRANKLIN, M. J., HELLERSTEIN, J. M. \& HONG, W. (2005) TinyDB: An Acquisitional Query Processing System for Sensor Networks. $A C M$ Transactions on Database Systems, 30(1), 122-173.

McNamara, K. \& Meynardi, A. (2010) Navigating the Road to Smart Grid: Modernizing the Critical Communication Infrastructure. Smart Grid. USA: Verizon.

Miao, G., Himayat, N., Li, Y. \& Swami, A. (2009) Crosslayer optimization for energy-efficient wireless communications: a survey. Wireless Communications and Mobile Computing, 9(4), 529-542.

Miller, J., Pullins, S. \& Bossart, S. (2008) " The Modern grid " U.S. Department of Energy, Office of Electricity Delivery and Energy Reliability. U.S. Department of Energy, Office of Electricity Delivery and Energy Reliability.

Naoto, K. \& Shahram, L. (2005) A survey on data compression in wireless sensor networks. In: Information Technology: Coding and Computing 2005. ITCC 2005. International Conference on, 4-6 April 2005 2005. IEEE Computer Society, 8-13.

Pourmirza, Z. \& Brooke, J. M. (2013a) Data Reduction for an ICT Network in the Smart Grid. In: The 9th International Conference on Intelligent Environments IE'13, 2013a Athens, Greece. IEEE Explore.

Pourmirza, Z. \& Brooke, J. M. (2013b) Monitoring and Visualising a Neighbourhood Area Sub-Grid. SMARTGREENS 2013-2nd International Conference on Smart Grids and Green IT Systems. Aachen, Germany: SCITEPRESS - Science and Technology Publications.

Pourmirza, Z. \& Brooke, J. M. (2013c) A Realistic ICT Network Design and Implementation in the Neighbourhood Area of the Smart Grid. Smart Grid and Renewable Energy, 4(6), 436-448.

Pourmirza, Z. \& Brooke, J. M. (2013d) The Wireless Sensor Network and Local Computational Unit in the Neighbourhood Area Network of the Smart Grid. In: International Conference on Sensor Networks (SENSORNETS), 2013d Barcelona, Spain. SCITEPRESS - Science and Technology Publications, 84-88.

Tan, H. O., Korpeoglu, I. \& Stojmenovic, I. (2007) A Distributed and Dynamic Data Gathering Protocol for Sensor Networks. Proceedings of the 21st International Conference on Advanced Networking and Applications. IEEE Computer Society.

Thangaraj, M. \& Ponmalar, P. P. (2011) A Survey on data aggregation techniques in wireless sensor networks. International Journal of Research and Reviews in Wireless Sensor Networks (IJRRWSN), 1(3), 36-42. 